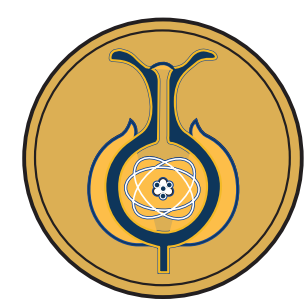

\title{
Criterios utilizados por profesores de matemáticas para el planteamiento de problemas en el aula
}

\section{Criteria used by mathematics teachers to pose problems in the classroom \\ Critérios utilizados pelos professores de matemática para a exposição dos problemas na sala de aula}

\section{Luis R. Pino-Fan}

luis.pino@ulagos.cl

Departamento de Ciencias Exactas

Universidad de Los Lagos

Osorno, Chile

Orcid: https://orcid.org/0000-0003-4060-7408

Juan G. Molina-Cabero

juangabrielmolinacabero@gmail.com

Colegio Eulogio Goicolea Garay

Calbuco, Chile

Orcid: https://orcid.org/0000-0002-6494-7100
Daniela Ilse Báez-Huaiquián

danielai.baezhuaiquian@gmail.com

Corporación SurEduca

Osorno, Chile

Orcid: https://orcid.org/0000-0002-1734-0912

\author{
Elizabeth Hernández-Arredondo \\ elizabeth.hernandez@ulagos.cl \\ Departamento de Ciencias Exactas \\ Universidad de Los Lagos \\ Osorno, Chile
}

Orcid: https://orcid.org/0000-0002-5285-1603

Received: $30 / O c t / 2019 \bullet$ Accepted: 5/feb/2020 • Published: 31/jul/2020.

\section{Resumen}

El estudio sobre los problemas que plantean profesores a sus estudiantes y las características que se deben considerar con la finalidad de potenciar sus competencias matemáticas constituye un tema que ha adquirido interés en los últimos años. Sin embargo, la mayoría de los estudios se han abordado desde el punto de vista epistémico, tecnológico o cognitivo, sin considerar efectivamente el quehacer que realizan los profesores habitualmente con respecto a la proposición de problemas para sus clases de matemáticas. En este sentido, el objetivo de este artículo es explorar las concepciones y prácticas pedagógicas de un grupo de docentes en servicio, de enseñanza básica y media del sur de Chile, sobre la selección de problemas de matemáticas para sus clases y los criterios que utilizan para tal selección. El interés de este estudio nace de asumir que, en el salón de clases, los profesores son el vehículo articulador de las políticas educativas; otra motivación surge por los resultados de la investigación en didáctica de la matemática. Con esta finalidad, se implementa un cuestionario, cuyos resultados evidencian que los problemas que eligen los profesores para sus clases son, básicamente, de tres tipos: i) problemas de los libros de texto, internet y otros recursos; ii) problemas que adaptan (variaciones propias) de los libros de texto, internet u otros 
recursos; y iii) problemas que el mismo docente crea. En cualquier caso, se hace notorio un creciente interés de los profesores por llevar al aula problemas con contextos cercanos a sus estudiantes y que despierten, emocionalmente, el interés. Además, se observó que los criterios utilizados para el planteamiento de problemas en el aula contemplan, implícitamente, los criterios de idoneidad propuestos en el marco del enfoque ontosemiótico del conocimiento y la instrucción matemáticos.

Palabras clave: Formación de profesores; matemáticas; resolución de problemas; diseño; educación matemática; profesores de matemáticas.

\section{Abstract}

The study of the problems posed by teachers to their students and the characteristics that should be considered to enhance mathematical competences is an issue that has gained interest in recent years. However, most of these studies have been approached from an epistemic, technological, or cognitive point of view, without effectively considering what math teachers usually do to pose problems in their classes. In this sense, the objective of this article is to explore the pedagogical conceptions and practices of a group of elementary and secondary teachers from southern Chile about the selection of mathematical problems for their classes, and the criteria they use for such selection. The interest of this study stems from assuming that, in the classroom, teachers are the articulating vehicle of educational policies. Another motivation for this paper is the results of research in Mathematics Education. For this purpose, a questionnaire was presented which showed that teachers choose any of the following three mathematical problems: i) problems taken from textbooks, internet, and other resources; ii) problems adapted from textbooks, internet, or other resources (own variations); and iii) problems created by the teacher. In any case, there is a growing interest in teachers to pose problems with contexts that students feel close to and arouse interest from an emotional point of view. In addition, it was observed that the criteria used by teachers for posing math problems in the classroom implicitly contemplate the didactical suitability criteria proposed within the framework of the Onto-Semiotic Approach to Mathematical Knowledge and Instruction (OSA).

Keywords: Teacher education; mathematics; problem solving; design; mathematics education; mathematics teachers.

\section{Resumo}

0 estudo dos problemas expostos pelos professores a seus alunos e das características que devem ser consideradas para aprimorar suas habilidades matemáticas é uma questão que tem despertado interesse nos últimos anos. No entanto, a maioria dos estudos foi abordada do ponto de vista epistêmico, tecnológico ou cognitivo, sem considerar efetivamente a tarefa que os professores costumam fazer com relação à proposta de problemas para suas aulas de matemática. Nesse sentido, o objetivo deste artigo é explorar as concepções e práticas pedagógicas de um grupo de professores em serviço, do ensino fundamental e médio do sul do Chile, sobre a seleção de problemas de matemática para suas aulas e os critérios utilizados para essa seleção. 0 interesse deste estudo decorre da suposição de que, na sala de aula, os professores são o veículo articulador das políticas educacionais; outra motivação surge dos resultados de pesquisas no ensino da matemática. Para isso, é implementado um questionário, cujos resultados mostram que os problemas eleitos pelos professores para suas aulas são basicamente de três tipos: i) problemas de livros didáticos, internet e outros recursos; ii) problemas que se adaptam (variações próprias) de livros didático, 
internet ou outros recursos; e iii) problemas que o próprio professor cria. De qualquer forma, há um crescente interesse dos professores em trazer problemas para a sala de aula com contextos próximos aos alunos e que despertem, emocionalmente, o interesse. Além disso, observou-se que os critérios utilizados para a exposição de problemas na sala de aula contemplam implicitamente os critérios de idoneidade propostos no âmbito do Enfoque Ontosemiótico do Conhecimento e a Instrução Matemática.

Palavras-chaves: Formação de professores; matemática; resolução de problemas; design; educação matemática; professores de matemática.

\section{Antecedentes}

Una de las líneas de investigación que ha sido intensamente estudiada por la comunidad internacional de investigación sobre didáctica de la matemática, es la resolución de problemas (Problem Solving), la cual explora características cognitivas, afectivas y actitudinales de los estudiantes cuando resuelven problemas de matemáticas (Felmer, Pehkonen \& Kilpatrick, 2016; Liljedahl, Santos-Trigo, Malaspina \& Bruder, 2016). Sin embargo, poco énfasis se le ha dado al estudio de cómo los profesores hacen el planteamiento de esos problemas, o sobre qué competencias y conocimientos requieren para el planteamiento o diseño de estos mismos (Singer, Ellerton, \& Cai, 2015), aun cuando el profesor es el vehículo que hace tangible el currículo y las propuestas educativas a partir de su diseño de clase -dentro de lo que se contempla el planteamiento de problemas- (Foster \& Inglis, 2017).

En el contexto latinoamericano, diversas iniciativas han surgido en los países con el fin de retomar la propuesta de Polya (1986) y Schoenfeld (1985) para potenciar los aprendizajes sobre matemáticas de los estudiantes, con base en la resolución de problemas. En particular, en Chile, diversas iniciativas han surgido lideradas, principalmente, por el grupo del Centro de Modelamiento Matemático (Felmer \& Perdomo,
2016; Perdomo, Felmer, Randolph \& González, 2017).

De tales propuestas, se evidencia que la resolución de problemas y el planteamiento de problemas deben ser vistos como complementarios. Por ejemplo, la National Council of Teachers of Mathematics (NCTM, 1989), ya recomendaba a los profesores brindar oportunidades para que los estudiantes piensen matemáticamente y desarrollen sus conocimientos mediante la creación de problemas en los procesos de enseñanza y aprendizaje. Halmos (1980), señalaba:

Los problemas son el corazón de las matemáticas, y espero que, como profesores, en las clases, seminarios, en los libros y artículos que escribimos, lo enfaticemos cada vez más, y capacitemos a nuestros estudiantes para que sean mejores proponedores de problemas y solucionadores de problemas que nosotros. (p. 524)

En este sentido, diversos autores sugieren que el profesor debe jugar un papel relevante de cara al planteamiento y resolución de problemas en el aula (Crespo, 2003). Malaspina, Mallart y Font (2015) señalan que es esencial que los docentes desarrollemos la competencia de crear problemas de matemáticas para poder orientar 
adecuadamente el desarrollo de tal capacidad en nuestros alumnos, y así puedan aprender matemáticas no solo resolviendo, sino también creando problemas. Malaspina (2016) lo plantea de la siguiente manera:

Consideramos esencial que los profesores desarrollen su capacidad de crear problemas, pues así podrán no sólo crearlos para proponer a sus alumnos problemas que respondan a la realidad y las motivaciones de ellos, sino que podrán también estimular a que sus alumnos aprendan creando, resolviendo y reflexionando problemas creados por ellos mismos. (p. 322)

Al respecto, la NCTM (2016) señala:

Para garantizar que los alumnos tengan la oportunidad de comprometerse con un pensamiento de alto nivel, los docentes deben seleccionar e implementar en forma regular tareas que estimulen el razonamiento y la resolución de problemas. Dichas tareas alientan el razonamiento y el acceso a las matemáticas mediante diversas formas de abordar los problemas, que incluyen la utilización de variadas representaciones y herramientas, así como la resolución de problemas a través de diferentes estrategias de solución. Es más, los docentes eficaces comprenden la forma en que pueden emplearse los contextos, la cultura, las condiciones y el lenguaje con el objeto de crear tareas matemáticas que traigan a colación el conocimiento previo y las experiencias anteriores de los estudiantes. (p. 18)
Como sugiere Malaspina (2017), la creación de problemas no debería verse como tarea exclusiva de los expertos, y se debe romper con la concepción de que los problemas a trabajar en clases son únicamente los que figuran en los libros de texto o en internet. Crear problemas es parte fundamental de la tarea docente. Cada profesor conoce la realidad específica en su aula, el entorno sociocultural y las motivaciones de sus alumnos y es un desafío a sus conocimientos y competencias didáctico-matemáticas, tanto crear secuencias de actividades como problemas adecuados para esa realidad.

En este sentido, el objetivo de este artículo es explorar las concepciones y prácticas pedagógicas de un grupo de profesores del sur de Chile, respecto del planteamiento de problemas de matemáticas en sus clases. Concretamente, nos interesamos en la caracterización de los criterios que consideran los profesores esenciales, al momento de elegir los problemas matemáticos que llevan al aula. Los resultados evidencian que, en sus clases, los profesores plantean los siguientes tipos de problemas: i) problemas de los libros de texto, internet y otros recursos; ii) problemas que adaptan (modificaciones/variaciones propias) de los libros de texto o de internet; y iii) problemas que ellos mismos crean.

\section{Marco teórico}

En la bibliografía científica, diversas posturas se han presentado sobre lo que se entiende por "problema" (Arcavi \& Frielander, 2007; Borasi, 1986; De Guzmán, 1993; Kilpatrick, 1985; Polya, 1986; Schoenfeld, 1985). Sin embargo, la mayoría de tales posturas describen lo que entienden por "problema matemático" a través de sus características, sin precisar una definición. 
Por tanto, desde esta perspectiva, definir lo que se entiende por creación de problemas es todo un reto.

Malaspina (2017) sugiere que, para precisar lo que se entiende por problema y por creación de problemas, es importante explicitar los elementos fundamentales de los problemas, y propone cuatro (p. 4):

- Información: datos cuantitativos o relacionales que se dan en el problema.

- $\quad$ Requerimiento: lo que se pide que se encuentre, examine o concluya, que puede ser cuantitativo o cualitativo, incluyendo gráficos y demostraciones.

- Contexto: puede ser intra matemático o extra matemático.

- Entorno matemático: el marco matemático global en el que se ubican los conceptos matemáticos que intervienen o pueden intervenir para resolver el problema (por ejemplo: funciones lineales; teoría de números; geometría analítica; cálculo diferencial; etc.).

A partir de lo anterior, parece lógico pensar en el aparente consenso de la comunidad internacional de investigación sobre educación matemática, respecto a que un problema es una situación o tarea para cuya solución no existe algoritmo o estrategia directa y exige una alta demanda cognitiva por parte de los sujetos que lo resuelven.

En consecuencia, la creación de problemas de matemáticas "es un proceso mediante el cual se obtiene un nuevo problema, por variación de un problema dado; o por elaboración (libre, a partir de una situación dada o configurada; o por un pedido específico, con énfasis matemático o didáctico)" (Malaspina, 2017, p. 4). Este mismo autor señala que la creación de un problema por variación se da cuando se modifica uno o más de los cuatro elementos fundamentales del problema.

Por otra parte, para el desarrollo del estudio utilizamos también algunas herramientas teórico-metodológicas del enfoque onto-semiótico (EOS) del conocimiento y la instrucción matemáticas (Godino, Batanero \& Font, 2007; 2019). Este enfoque teórico surge al seno de la educación matemática, con el objetivo de articular las diversas dimensiones -epistémicas, cognitivas, afectivas, interaccionales, mediacionales y ecológicas- involucradas en los procesos de enseñanza y aprendizaje de las matemáticas. A nivel internacional, el EOS se presenta como un sistema teórico inclusivo que trata de articular diversas aproximaciones y modelos teóricos utilizados en la investigación en educación matemática (Kaiber, Lemos \& Pino-Fan, 2017; Presmeg, 2014).

De particular interés para este estudio es la noción de sistemas de prácticas (o prácticas matemáticas), pues juega un rol fundamental en el EOS, tanto desde la perspectiva epistemológica como la didáctica. Para Font, Godino y Gallardo (2013), las prácticas matemáticas se pueden definir como la combinación de una práctica operativa, a través de la cual se pueden leer y producir textos matemáticos, y una práctica discursiva, que permite reflexionar sobre las prácticas operativas. Estas prácticas pueden ser llevadas a cabo por una persona (sistema de prácticas personales) o compartidas dentro de una institución (sistema de prácticas institucionales).

Así, en las prácticas matemáticas que realiza una persona o institución, con el propósito de resolver situaciones-problemas, intervienen objetos ostensivos (símbolos, gráficos, etc.) y no ostensivos (conceptos, proposiciones, etc.), que evocamos al hacer matemáticas y que son representados en forma textual, oral, gráfica o incluso gestual. Es 
decir, las situaciones-problema son el origen o razón de ser de la actividad matemática; con el lenguaje se representa las restantes entidades y sirve de instrumento para la acción; los argumentos justifican los procedimientos y proposiciones que relacionan los conceptos entre sí. Estos objetos matemáticos primarios (situaciones/problema, elementos lingüísticos, conceptos/definiciones, propiedades/proposiciones, procedimientos y argumentos) se articulan para configurar la práctica matemática. A tal articulación en el EOS se le conoce como configuración de objetos matemáticos primarios. Estas configuraciones pueden ser socio-epistémicas (redes de objetos institucionales) o cognitivas (redes de objetos personales), según si los objetos matemáticos primarios se ponen en juego en prácticas institucionales o personales, respectivamente.

En el marco del EOS, también se ha propuesto un modelo de conocimientos y competencias didáctico-matemáticas del profesor -modelo CCDM- (Pino-Fan, Font \& Breda, 2017; Godino, Giacomone, Font \& Pino-Fan, 2018). Este modelo establece, entre otras cosas, que los conocimientos del profesor de matemáticas se pueden categorizar en tres grandes dimensiones: matemática; didáctica; y meta didáctico-matemática (Pino-Fan, Assis \& Castro, 2015; Pino-Fan \& Godino, 2015). Además, sugiere que las competencias clave del profesor de matemáticas son dos: competencia matemática y competencia de análisis e intervención didáctica; esta última tiene como núcleo fundamental diseñar, aplicar y valorar las secuencias de aprendizaje tanto propias como las de otros, por medio de técnicas de análisis didáctico y criterios de calidad para establecer ciclos de planificación, implementación, valoración y planteamiento de propuestas de mejora (Breda, Pino-Fan
\& Font, 2017; Font, 2011). Mientras que la competencia matemática se relaciona con la resolución de problemas (a priori de la implementación), el análisis de los objetos matemáticos primarios que se ponen en juego con un determinado problema (para asegurar que los contenidos tocados con el problema estén acordes con los objetivos que se pretenden en el aula), el diseño de tareas o planteamiento de problemas propiamente tal, y el análisis de la actividad matemática desarrollada por los estudiantes (análisis de prácticas personales).

En el modelo CCDM se asume que las herramientas teóricas del EOS permiten el desarrollo de las competencias clave del profesor de matemáticas, por ejemplo, mediante análisis en términos de prácticas, configuración de objetos y procesos matemáticos (y didácticos). Por ello se recomienda que el profesor conozca y comprenda la idea de configuración de objetos y procesos activados en una determinada práctica matemática y sea capaz de usarlos de manera competente en los procesos de enseñanza y aprendizaje de las matemáticas (Pino-Fan, Godino y Font, 2018) y esto, a su vez, le permitirá utilizarlos como aspectos a considerar al momento de planificar o crear sus problemas.

\section{Metodología}

El paradigma en el que se inscribe nuestra investigación, es el cualitativo (Cohen, Manion \& Morrison, 2011), dado que, por un lado, se planificó e implementó un cuestionario para explorar los criterios que comúnmente tienen presentes los profesores al momento de elegir (crear o seleccionar) sus problemas matemáticos para el aula. Y, por otro lado, se diseñaron entrevistas semiestructuradas para profundizar en los aspectos señalados por los profesores sobre el tema. 


\section{Sujetos y contexto}

Los sujetos del presente estudio son 11 profesores de enseñanza media en activo, todos ellos con experiencia mínima de 3 años en aula y, además, al momento de llevar a cabo el estudio, todos cursaban el segundo semestre del Magíster y del Doctorado en Educación Matemática de la Universidad de Los Lagos. Todos ellos, en algún momento de su carrera, habían dictado clases sobre alguno de los tópicos matemáticos presentes en el currículo chileno de enseñanza media.

Para el estudio, se utilizó una muestra intencionada (no probabilística), la cual se refiere a que los sujetos de la muestra, no fueron elegidos al azar, sino de forma intencional. En concreto, se trata de profesores que voluntariamente decidieron participar en nuestro estudio una vez que se les informó el objetivo. La aplicación del cuestionario se efectuó sobre el total de la muestra (11 profesores), de los cuales se seleccionan 7 para la fase de entrevistas. Los cinco profesores entrevistados se eligieron según las 3 categorías identificadas: creación de problemas por variación (3 profesores), creación de problemas por elaboración (3 profesores) y selección de problemas (1 profesor).

\section{Instrumentos para la recolección de datos}

Para la recolección de los datos se pretendía diseñar un test o cuestionario, para lo cual se hicieron versiones preliminares. Sin embargo, nos percatamos que estas versiones preliminares eran muy próximas al test diseñado por Klinshtern, Koichu y Berman (2015), para su estudio sobre las concepciones de 150 profesores sobre el planteamiento de problemas. Por lo tanto, dado que se ajustaba a nuestros objetivos, nos pareció pertinente utilizar dicho instrumento (Klinshtern, Koichu y Berman, 2015, p. 464).

Para ello, el instrumento se tradujo al español, y posteriormente se adaptó al contexto chileno, mediante la introducción de algunos cambios en la forma de expresar las preguntas y otros detalles de redacción y forma. Además, se omitieron algunas preguntas sobre los detalles personales de los sujetos (e.g., e-mail, teléfono, etc.). El cuestionario resultante, el cual finalmente se aplicó a nuestra muestra de profesores, se presenta como Anexo de este artículo.

Como se señaló anteriormente, la investigación tuvo dos fases claramente identificadas en lo relativo a la recogida de la información: aplicación del cuestionario y entrevistas semiestructuradas. En este sentido, es importante destacar que el cuestionario, aplicado en la primera fase, tuvo por objetivo determinar cómo los profesores seleccionaban los problemas que consideraban en sus planificaciones y que finalmente llevaban al aula, resultando en tres categorías de selección de problemas. Esta primera categorización permitió diseñar las entrevistas.

Se prepararon tres guiones para entrevistas semiestructuradas, uno por cada categoría encontrada como resultado del análisis de las respuestas al cuestionario, sobre el tipo de problemas que eligen los profesores para llevar al aula: creación de problemas por variación, creación de problemas por elaboración y selección de problemas. Con las entrevistas nos desmarcábamos del estudio de Klinshtern, Koichu y Berman (2015), pues el énfasis que se le dio fue la indagación de los aspectos/criterios que utilizaban los profesores para crear o seleccionar problemas para el aula. En la sección de análisis, se evidencia el tipo de preguntas que se realizaron a los profesores en las entrevistas. Las entrevistas tuvieron una duración de 40 minutos, y 
todas fueron grabadas con cámara de vídeo y grabadoras de audio. Entre un entrevistado y otro, los primeros tres autores de este documento daban un espacio de 20 minutos, tiempo que sirvió para tomar apuntes personales sobre la entrevista que acababa de terminar, y compartir (triangular) algunas opiniones con el resto del equipo.

\section{Herramientas para el análisis de los datos}

Además de los aspectos descritos en la sección de marco teórico -concretamente, la propuesta de Malaspina (2017) sobre la creación de problemas, y las nociones de prácticas matemáticas y configuración de objetos matemáticos primarios del EOS-, se utilizaron los criterios de idoneidad didáctica del modelo CCDM y del EOS (Breda, Font \& Pino-Fan, 2018; Breda, Pino-Fan \& Font, 2017; Font, Planas \& Godino, 2010), los cuales permiten identificar y valorar aspectos matemáticos didácticos de los procesos de estudio $\mathrm{y}$, concretamente, de los problemas que diseñan, implementan y evalúan los profesores. Estos criterios (con sus respectivos descriptores) refieren a las siguientes seis idoneidades: 1) epistémica, para valorar si las matemáticas que están siendo enseñadas son "buenas matemáticas"; 2) cognitiva, para valorar, antes de iniciar el proceso de instrucción, si lo que se quiere enseñar está a una distancia razonable de aquello que los alumnos saben, y después del proceso, si los aprendizajes adquiridos están cerca de aquello que se pretendía enseñar; 3) interaccional, para valorar si las interacciones resuelven dudas y dificultades de los alumnos; 4) mediacional, para valorar la adecuación de los recursos materiales y temporales utilizados en el proceso de instrucción; 5) emocional (o afectiva), para valorar la implicación (intereses, motivaciones...) de los alumnos durante el proceso de instrucción; y 6) ecológica, para valorar la adecuación del proceso de instrucción al proyecto educativo del centro, las directrices curriculares, las condiciones del entorno social y profesional (Font, Planas \& Godino, 2010).

\section{Análisis y discusión de los datos}

A continuación, se presenta el análisis y la discusión de los resultados de nuestro estudio; para ello, se divide la presentación en dos momentos: resultados y discusión de la aplicación del cuestionario; resultados y discusión de las entrevistas.

\section{Resultados de la implementación del cuestionario}

Respecto de la pregunta 1 del cuestionario (Anexo 1), identificamos que, aunque los participantes del estudio de manera preliminar señalaron trabajar en enseñanza media, en la actualidad ellos se desempeñan en el nivel universitario -tanto en pregrado como en postgrado-, en Enseñanza Media y también en Enseñanza Básica. Este dato es de interés, ya que, como señalan Ron, Zaslavsky y Zodik (2013), el diseño de la tarea está directamente influenciado por el objetivo, el cual estará ligado a cada nivel educativo. Por lo tanto, los problemas dependerán en gran parte del contexto en el que se utilizarán. Es decir, el nivel educativo en el que forjan sus experiencias los profesores influye en la decisión que toman.

Respecto de la pregunta dos, años de experiencia, se encontró que seis (6) de los once (11) participantes del estudio, poseen al menos 10 años de experiencia en el aula, y solo dos (2) poco más de 2 años. Un punto relevante de la experiencia de los docentes es 
que, en la realidad del contexto chileno, ellos pueden ejercer en más de un nivel educativo durante su carrera (básica, media, universitario...), lo cual les genera experiencias y aprendizajes en variados contextos, aspecto que también podría determinar sus decisiones al momento de plantear problemas.

En relación con la pregunta tres, el nivel de complejidad de sus clases, ocho profesores declaran que el nivel de complejidad de las tareas/problemas que proponen en clases es de nivel medio, mientras que el resto de los profesores declara que el nivel de sus problemas es alto. No obstante, a partir del cuestionario no se evidencian cuáles son los aspectos que, según los profesores, determinan tal complejidad. Entre los factores mencionados por los docentes en la entrevista que se analizará más adelante, sobre el cómo se determina la complejidad de los problemas utilizados, se observa que prefieren aumentar la complejidad gradualmente, en otro caso mencionan que tal complejidad dependerá del nivel de conocimiento matemático que posee cada curso (conocimientos previos) o cada alumno en particular.

Respecto del tipo de recursos $-\mathrm{y}$ su frecuencia-que utilizan los profesores para seleccionar problemas matemáticos útiles para su práctica de enseñanza (pregunta 4), se encontró que los libros de texto escolares son el recurso todavía más utilizado ( 5 casi siempre; 4 a menudo; 2 a veces). Love y Pimm (1996) señalan, al respecto, que "el libro es todavía la tecnología más extendida y usada en las clases de matemáticas.... el libro de texto ha moldeado nuestra noción de la matemática y como debe enseñarse" (p. 402). Después de los libros de texto, los recursos más frecuentemente utilizados por los profesores fueron "otros libros" ( 4 casi siempre; 4 a menudo; 2 a veces; 1 raramente) y "estudios académicos anteriores -notas, apuntes, etc.--" (4 casi siempre; 3 a menudo; 2 a veces; 1 raramente; 1 casi nunca). Mientras que los recursos menos utilizados para la selección de problemas fueron "problemas planteados por los alumnos" (5 raramente; 6 casi nunca) y "plantear mis propios problemas" ( 2 casi siempre; 2 a menudo; 6 raramente; 1 casi nunca). Lo anterior evidencia que, aunque el uso de problemas creados por los profesores está presente en el aula, el uso de problemas extraídos fielmente de los textos es mucho más utilizada y, además, raramente contemplan para sus clases el uso de problemas planteados por los estudiantes.

De forma complementaria, la pregunta 5 que explora la frecuencia de uso, en las prácticas pedagógicas de los profesores, de los problemas creados por los docentes o los alumnos, la discusión en clase y trabajo en grupo, tuvo como resultado lo siguiente: la mayoría de los participantes (7 casi siempre y 4 a menudo) promueven la discusión en sus clases; también se fomenta el trabajo en grupo (4 casi siempre, 5 a menudo, 2 a veces); solo 2 profesores señalan que casi siempre utilizan sus propios problemas ( $4 \mathrm{a}$ menudo, 3 a veces, 2 raramente); y únicamente un profesor motiva a sus estudiantes a plantear sus propios problemas ( 3 a menudo, 5 a veces, 2 raramente).

Por su parte, la pregunta 6 , sobre la finalidad para plantear sus propios problemas, permitió identificar que existen tres motivos más recurrentes por los cuales los profesores eligen crear sus propios problemas (por variación o elaboración): 1) la necesidad de que el problema se adecue al contexto determinado de la clase; 2) que el problema sea de interés para los estudiantes; y 3 ) la necesidad de dar variadas aplicaciones a un contenido. La Tabla 1 muestra algunas de las respuestas a la pregunta y el propósito para el planteamiento de problemas que se desprende de la respuesta. 
Tabla 1

Propósito de los profesores al plantear sus propios problemas

Respuesta

Motivación

Lo hago porque trato de contextualizar lo que hago en clases con el nivel sociocultural gel contecto faniliair

Con el propónito de lograr aprendizajes que tean

Adecuación del

contexto

Fra tener el control de los respuestos espe
rodas en cado problema.

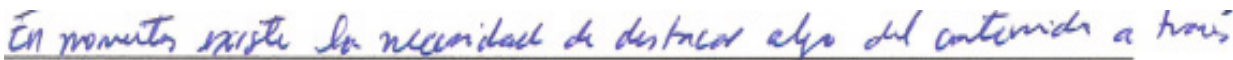

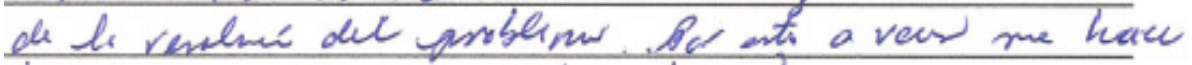
biew es und min popín problima

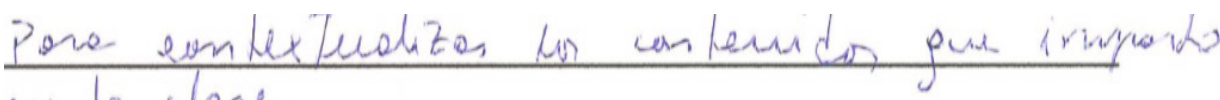
en le close

10. hago porque para cear estos problemas considero las

Caractensticas de mis erles diantes y de esta manela

alercar la matematica a ellos. Esto hau que particiem más y tengan una mya disposición.

Plantieo mis propos problemas porque se me ocurien maneras interssantes (desde mi pernte de vista) de introducic o explicar deternuniado concefoto, O para aelacional diferende registios, ete. Porque se recuiere adaptar las problematices y uiveles al ritur y capacidads de tapuendizaje de ln estudiants - Para plantéar la resolución de problemas y que los Se va de lo más foucil a lo más complejo, to - Lueg se lleva todos los conocimintos a li

Control de resultados

Aplicación de contenidos

Adecuación del contexto

Adecuación del contexto

Problemas de interés para estudiantes

Adecuación del contexto

Aplicación de contenidos veda cotediana, para que asi lo relacionen.

Nota: Fuente propia de la investigación.

Respecto de las responsabilidades que tienen los profesores, además de impartir sus clases, en las instituciones en las que laboran (pregunta 7), los 11 profesores señalaron que seleccionan los problemas que utilizarán en sus clases, los 11 planifican cada una de sus clases, los 11 son responsables de ejecutar sus planificaciones (impartir sus clases), 9 son responsables de la planificación del año escolar. Esta información es 
de relevancia en la medida que nos permitirá realizar un contraste en cuanto al tiempo que disponen los profesores para crear e implementar problemas, y sobre cómo esto influye o no al momento de decidir si crear sus propios problemas o seleccionarlos de los libros de texto.

\section{Las entrevistas}

Como se mencionó, a partir de los resultados del cuestionario se identificaron tres categorías de profesores, en relación con los problemas que proponen en sus clases, a saber: $i)$ los que crean sus propios problemas (creación de problemas por elaboración); ii) los que toman problemas creados y los modifican o adaptan (creación de problemas por variación); y iii) los que seleccionan de distintas fuentes problemas ya creados. Las entrevistas tuvieron por finalidad profundizar en los criterios que tienen presentes, de forma explícita o implícita, los profesores de cada una de las tres categorías, para seleccionar sus problemas para sus clases de matemáticas. A continuación, presentamos los análisis de las entrevistas realizadas a los profesores, por categoría.

\section{Profesores que crean problemas por elaboración}

La entrevista se centró, principalmente, en 4 aspectos: 1) Motivación para crear problemas; 2) Criterios considerados en la creación de problemas; 3) Elementos que han forjado los criterios utilizados; y 4) Dificultades que se presentan en la creación de problemas.

Sobre el primer aspecto, los profesores entrevistados señalaron que prefieren la creación de problemas al momento de planificar sus clases, debido a la falta de problemas que fomenten la reflexión matemática y la necesidad de abarcar diferentes contextos en los cuales se sitúen los estudiantes. Tal aspecto se muestra por ejemplo en la siguiente respuesta: "Para mí, es importante que hagan esto [fomenten la reflexión matemática y abarquen diferentes contextos] y bueno, diseñar problemas que estén en un contexto real, con datos reales" (Profesor 1). Además, hacen mención a la falta de problemas aplicados en los libros de texto y otros materiales que proporcionan sus instituciones, el Profesor 2 lo señala de la siguiente forma: “... [la creación de problemas] tiene que ver con algo más bien personal, a mí siempre me pareció que, si en la matemática no se resolvían problemas reales, no tenían utilidad".

En general, podemos decir que los factores o motivos principales que llevan a los profesores a crear sus propios problemas al momento de planificar sus clases de matemáticas fueron aspectos de tipo epistémico, cognitivos y ecológicos de la didáctica de la matemática, relacionados con la falta de problemas específicos que se adapten a un grupo determinado de estudiantes con características particulares, con los cuales se promueva la reflexión matemática y considere el contexto de los estudiantes.

Sobre el segundo foco de la entrevista, criterios para creación de problemas, los profesores entrevistados consideran que los criterios aplicados para la creación de problemas matemáticos, son de gran importancia, ya que los problemas deben ser "atractivos para los estudiantes", generando pertinencia y coherencia entre la tarea, la demanda cognitiva que exige la misma por parte de los estudiantes, y el procedimiento que se espera que desarrollen los estudiantes. Además, enfatizan la importancia de crear problemas con los cuales se cree sentido y utilidad de las matemáticas que se 
están utilizando (para resolver el problema), y no solo problemas en los que se "apliquen por aplicar contenidos matemáticos”. El profesor 1 señala al respecto: "Que sean problemas de intervención, es decir que no utilicen un solo tipo de problemas para resolverse, sino que sean problemas un poco más complejos que requieran el apoyo de diversos conceptos matemáticos".

Otro criterio señalado por los profesores, como ya lo señalamos antes, es el contexto en el que realizan su práctica el profesor y los estudiantes, de manera que permita que la reflexión de los contenidos matemáticos no sean solo en términos intra-matemáticos sino también en situaciones reales; esto es, que permitan relacionar contenidos y significados de tales contenidos. El profesor 2 señala al respecto: "El tema del contexto era uno, el tema de la estructura de matematización era otro, ...ojalá partiera de situaciones reales de los estudiantes".

Sobre el tercer foco de la entrevista, elementos que han permitido considerar o forjar criterios utilizados para la creación de problemas, los profesores señalan que los han adquirido mayoritariamente a través de los años de experiencia en su práctica profesional $\mathrm{y}$, en menor medida, como parte de su formación universitaria. El Profesor 3 señala:

“...cuando tuve mayor acercamiento a qué cosas debería tener un problema... yo creo que durante la marcha, con el tiempo, uno va viendo cuáles son las cosas que más le interesan a los estudiantes".

Por su parte el Profesor 1 comenta:

“... las metodologías de los últimos años de la universidad, proporcionan algunos aspectos, aunque a veces no se pueden considerar en contextos reales. Por eso yo aprendi a crear problemas mirando lo que existía en los textos en esos años, después sigo la propuesta de la Reforma del 2000 y después de la Reforma del 2000 a la adhesión con las exigencias de las mediciones SIMCE”"

El Profesor 2 señaló que, para formarse unos criterios para plantear problemas, tuvo que ser autodidacta y "observador" de los textos de otros países y contextos, y hace un llamado, que se evidencia con las respuestas de los otros profesores encuestados, sobre la necesidad de espacios para la formación de competencias (didáctico-matemáticas) que permitan la realización de actividades profesionales clave para el docente, tal como el planteamiento de problemas. El Profesor 2 comenta: "...aparte de revisar toda la bibliografia nacional también, revisaba textos que habian sido editados en Estados Unidos, otros que habian sido editados en Francia, de todo esto se hace una síntesis y luego empezar a estudiar los ejercicios". El Profesor 3, señala sobre el tema: “...yo nunca he dejado de estudiar matemáticas, siempre he estado estudiando matemáticas de forma autodidacta, entonces yo creo que si ha facilitado, también los procesos de aplicación".

En síntesis, de este tercer foco se desprende la necesidad de que en la formación docente existan asignaturas (o espacios) donde el profesor se relacione con el tema $\mathrm{y}$ pueda tener variadas formas (y experiencias) de enseñar un contenido matemático (ver por ejemplo las recomendaciones de Pino-Fan, Guzmán, Larraín \& Vargas, 2018; Pino-Fan, Godino \& Font, 2015), formación que complemente la experiencia que el docente va forjando con el tiempo. 
Finalmente, sobre el cuarto foco de la entrevista, dificultades para la creación de problemas, los profesores señalan aspectos mediacionales (como el tiempo), epistémicos -relacionados con las competencias matemáticas de los docentes, para analizar la actividad matemática de los estudiantes y análisis de los problemas y prácticas matemáticas sugeridas por los textos-, así como competencias relacionadas con el análisis e intervención didáctica, que les permita identificar y comprender los factores que influyen en los procesos de enseñanza que implementan. Al respecto, el Profesor 2 señala: "El tiempo es una variable muy importante, pero más tiempo no garantiza nada, tiene que haber un desarrollo profesional, tiene que haber un desarrollo de competencia, o sea si el tipo no tiene la noción de crear problemas hay que enseñarle o capacitarlo". Por su parte el Profesor 3 comenta: “...llevar al aula problemas creados siempre... es como una expectativa, porque no podemos saber muy bien qué es lo que va a suceder, cuáles son todas las variables que van a intervenir, y a veces cuando es un problema de libro, cómo se puede analizar para saber si está bueno o malo".

El Profesor 1 declaró en la entrevista algo que ya había sido observado en el estudio de Malaspina, Mallart y Font (2015), sobre el impacto de la creación de problemas en la competencia matemática de los profesores; el Profesor 1 lo señala de la siguiente manera: "Yo creo que me ayudó a desarrollarme como profesor, me ayudó a desarrollarme cognitivamente, me ayudó a desarrollarme y comprender mejor la matemática que enseño".

\section{Profesores que crean problemas por variación}

Al igual que en la categoría anterior, la entrevista aquí se enfocó en cuatro aspectos: 1) Motivación para crear problemas; 2) Criterios considerados en la creación de problemas; 3) Elementos que han forjado los criterios utilizados; y 4) Dificultades que se presentan en la creación de problemas por variación.

En esta categoría, los profesores entrevistados señalaron que generalmente prefieren la adaptación de problemas (creación de problemas por variación), por sobre la creación de sus problemas por elaboración, debido a las exigencias de las instituciones en las que laboran, sobre los libros de textos establecidos por el reglamento. Advierten que, en relación con ello y como primer aspecto que los motiva a crear/variar problemas, es que los textos de las instituciones contienen problemas que no se adaptan a todas las necesidades de los estudiantes. Lo anterior se evidencia, por ejemplo, con la respuesta del Profesor 4, quien señala:

"La idea del colegio es que como el alumno compraba el libro, nosotros como profesores nos veíamos obligados a utilizarlo; entonces, a veces los problemas con los que me tocaba trabajar no cumplian con todas las expectativas, por lo cual, haciendo uso de los mismos problemas, los adaptaba a través de preguntas para que los alumnos de alguna manera desarrollaran más lo que estaba buscando”.

Además, los profesores mencionan que otro motivo para crear/variar problemas es la falta de problemas en los textos que desarrollen el razonamiento matemático, y no solo ejercicios en los cuales se apliquen fórmulas o se desarrollen algorítmicamente. El Profesor 5 comenta:

"...son problemas que surgen de modificar problemas que me encuentro, y yo se los traigo a los muchachos 
porque son problemas diferentes que pueden ser más interesantes, y lo otro que yo pienso, que puede ser interesante desde el punto de vista del razonamiento matemático, es que traigan [los problemas] un tipo de razonamiento nuevo, que no han visto los otros problemas triviales que suelen tener los libros".

Es decir, los profesores de esta categoría identifican y recolectan problemas de textos $\mathrm{u}$ otros recursos, los analizan y los adaptan. Dicha adaptación, para ellos, puede ser por tres motivos: a) obtener un problema que se adapte a un contexto más próximo al contexto de los estudiantes; b) obtener un problema que fomente más el razonamiento matemático; y 3 ) obtener problemas que sean más novedosos y de interés para los estudiantes.

Sobre el segundo foco de la entrevista, criterios considerados en la creación/variación de problemas, los profesores mencionan que los criterios a considerar no siempre deben comenzar por el aspecto puro de la matemática (representaciones, definiciones, teoremas, procedimientos), sino que estos deben estar relacionados con la afinidad entre los estudiantes y la matemática que se quiere enseñar. Es decir, considerar aspectos cognitivos (como los errores frecuentes de los estudiantes en el tema que se quiere estudiar), ecológicos (como los contextos y aspectos sociales), epistémicos (riqueza de la matemática y su interacción con otros aspectos como los recursos tecnológicos, contexto, etc.). El Profesor 4 señala al respecto:

"cuando yo hice toda mi carrera profesional, yo nunca vi didáctica, ni pedagogía, ni aspectos pedagógicos, ni nada de esto. Entonces mi idea era que las matemáticas siempre se tenían que diseñar desde el aspecto puro, la matemática propia y sus elementos, y cuando entre acá [magister en educación matemática], te das cuenta de que debes considerar aspectos tan olvidados como la afectividad que el estudiante tiene con la matemática, los aspectos cognitivos de los estudiantes, el contexto y su interacción con las matemáticas que se quieren ensenar, los recursos materiales y su interacción con las matemáticas, etc.”.

Además, se menciona que para que un problema adoptado tenga una buena aceptación por parte de los estudiantes, depende de una serie de conocimientos tanto matemáticos como didácticos, con la finalidad de promover el razonamiento matemático en los estudiantes. Como indica el Profesor 6: "Un criterio es que sea novedoso [el problema], desde el punto de vista del razonamiento matemático, y que además tenga afinidad con lo que hacen los estudiantes y el mundo en el que se encuentran insertos".

Como cierre del segundo punto de la entrevista, los profesores señalan que para crear/variar un problema y que este logre los objetivos planteados para una determinada clase, deben utilizarse criterios que surgen de un amplio trabajo autónomo por parte del docente, sea por que son "autodidactas" o porque han fomentado sus competencias matemáticas y didácticas mediante cursos de capacitación. Al respecto, el Profesor 4 señala: "los criterios para adaptar mis problemas tienen que ver con los aspectos de tus estudiantes como tal, depende de una serie de conocimientos, si así lo quieren ver, dimensiones del conocimiento del profesor, y por tanto la adaptación de mis problemas no depende de una sola cosa sino de una serie de cosas". 
Sobre el tercer foco de la entrevista, elementos que han permitido considerar o forjar criterios utilizados para la creación/ variación de problemas, al igual en la categoría de profesores que crean sus problemas por elaboración, en esta categoría los profesores señalan que la experiencia les ha permitido determinar tales criterios. El Profesor 5 lo señala: "Por la experiencia, nunca he recibido capacitación, hasta ahora que entré al magister. Espero recibir herramientas que complementen lo aprendido con mi experiencia".

Otro profesor señala que los criterios también se generaron o fortalecieron en cursos de capacitación o formación, tales como maestría en Matemática Educativa, y no en la formación pedagógica (universitaria) donde se profundizaron conocimientos didácticos. El Profesor 6 señala al respecto:

“... lo que tratas de hacer es que todo ese conocimiento que de alguna manera te brinda la formación en la maestría, cómo todo esto te permite tratar de hacer una adaptación, de llevarlo al aula de clases, hacerlo funcional, a través de todo esto de las consideraciones de los objetivos, es decir ¿Qué es mejor para mis estudiantes?".

Cabe destacar, a partir de las entrevistas con los profesores de esta categoría, que no necesariamente un docente se titula (de la universidad) sabiendo cómo se debe adaptar un problema matemático de forma correcta, tampoco una forma infalible de reconocer cuáles son los criterios más importantes para considerar. Este conocimiento, sobre los criterios, se relaciona con las competencias didácticas y matemáticas del profesor, y se van forjando tanto con la experiencia profesional del docente como con los cursos de formación/capacitación que recibe para fortalecer sus competencias profesionales. Es decir, se trata de un proceso que se comienza en la formación y se continúa perfeccionando con la experiencia que aumenta con el paso de los años.

Respecto del cuarto foco de la entrevista, dificultades para la creación/variación de problemas, nuevamente, al igual que en la categoría anterior, el factor tiempo sale a colación como principal dificultad tanto para planificar las clases y como para la implementación con problemas creados por variación que exigen una demanda cognitiva mayor a la exigida habitualmente por los textos. Como señala el Profesor 4, "el tiempo es un factor importante, porque tú tienes que aprender a gestionar cómo vas a distribuir el objeto matemático, y tener una idea de cuáles son las posibles soluciones que vayan a dar los estudiantes y si el contenido que vas a enseñar es el adecuado para los estudiantes".

Otra dificultad señalada por los profesores es la existencia de reglamentos institucionales que los obligan a utilizar problemas que se encuentran en los libros de texto que utiliza una determinada institución, los cuales, según ellos, no siempre se adaptan a las necesidades y contextos de los estudiantes. Por otro lado, y relacionado con el tiempo, los profesores también señalan el exceso de carga laboral del docente en Chile, ya que de algún modo dificultan la realización de planificaciones apropiadas que estén acordes al contexto en el cual los docentes y estudiantes llevan a cabo el proceso de estudio. El Profesor 5 lo manifiesta del siguiente modo: "El exceso de trabajo, está desde la enseñanza básica hasta la universidad, o sea ... básicamente, quieres hacer una educación de calidad, pero las responsabilidades no lo permiten, básicamente necesitamos vivir y hacer dinero". 
Otro punto señalado como dificultad, y que los profesores señalan que debe reflexionarse, es el "nivel cognitivo" que tienen los estudiantes para poder resolver problemas matemáticos (i.e., déficit de conocimientos previos requeridos), "ya que es una de las consideraciones principales que impide al docente formular o adaptar sus propios problemas que cumplan con las expectativas. Una de las principales dificultades es el hecho de cómo hacerlo uniforme porque cada alumno es diferente".

Profesores que seleccionan de distintas fuentes sus problemas

Al igual que en las dos subsecciones anteriores, la entrevista de esta categoría se enfocó a 4 aspectos:1) Motivación para seleccionar problemas ya creados; 2) Criterios considerados para la selección de problemas; 3) elementos que han forjado los criterios utilizados; y 4) Dificultades que se presentan para la selección de problemas ya creados.

En esta categoría se entrevistó al único profesor (Profesor 7) que señaló que para el diseño de sus clases se basa exclusivamente en la selección de problemas (ya creados) de textos y otras fuentes y recursos. Así, en relación con el primer punto abordado en la entrevista, menciona que prefiere la selección de problemas ya creados, concretamente para formar "guías de ejercicios", debido a los bajos niveles de aprendizaje que ha observado en sus estudiantes (conocimientos previos).

Así mismo, el profesor señaló el tiempo como factor que lo motiva a seleccionar problemas, pues al tener planes de estudio, acordes con el currículo nacional, muy densos en cuanto a todos los contenidos que tiene que enseñar en un período determinado, por lo cual no le queda tiempo para llevar a cabo el proceso de creación de problemas.
En cuanto al segundo foco de la entrevista, criterios que considera al momento de seleccionar sus problemas, el profesor considera fundamental tener en cuenta el contexto de los estudiantes, ya que el contexto permite, según el profesor, dar "aplicabilidad" a los contenidos matemáticos que se están estudiando. Él lo señala así: "los selecciono con respecto al contexto, o sea, depende del tipo de alumno al que le voy a enseñar. También veo que tanta matemática podrían utilizar ellos, entonces no tomo problemas que sean de mucha demostración, de matemática pura, sino problemas que son más aplicables para ellos".

Por otro lado, respecto de los elementos que han permitido considerar o forjar criterios utilizados para la selección de sus problemas, el profesor mencionó que no se le enseñó a seleccionar problemas durante su formación como profesor, y mucho menos tuvo espacios en los cuales adquiriera competencias que le permitieran plantear problemas. El profesor comenta al respecto: "En la universidad no se me enseñó cómo seleccionar los problemas matemáticos, porque en realidad mi carrera está más orientada a la modelación e investigación pedagógica". En consecuencia, el profesor continúa comentando que, los criterios que utiliza para la selección de problemas los adquirió por medio de la experiencia y el traspaso de conocimiento entre sus colegas.

Finalmente, respecto del último punto, las dificultades para seleccionar sus problemas, el profesor respondió más que dificultades, aspectos complementarios a sus respuestas sobre las motivaciones para dedicarse a la selección de problemas. Así, por ejemplo, enfatizó que existe una falta de tiempo para desarrollar las clases y a esto, según él, se le suma los bajos niveles de conocimiento matemático que poseen los estudiantes (conocimientos 
previos requeridos), lo que hace de este proceso algo aún más extenso en cuanto a tiempos, pues se tendrían primero que instalar conocimientos previos requeridos.

\section{Reflexiones finales}

En este artículo nos propusimos explorar cómo los profesores seleccionan los problemas para sus clases de matemáticas y cuáles son los criterios que consideran para hacer la selección de un problema determinado. Así, a partir de los resultados del cuestionario se identificaron tres categorías de profesores, con relación a los problemas que proponen en sus clases, a saber: i) los que crean sus propios problemas (creación de problemas por elaboración); ii) los que toman problemas creados y los modifican o adaptan (creación de problemas por variación); y iii) los que seleccionan de distintas fuentes problemas ya creados.

A partir de esta categorización, se realizaron entrevistas semiestructuradas a profesores de cada categoría, con la finalidad de determinar cuáles eran los criterios que tenían presentes en cada caso (Tabla 2).

Un aspecto que debemos resaltar del discurso de los profesores, cuando respondían preguntas sobre los criterios o aspectos que consideraban al elegir sus problemas

matemáticos para el aula, es que los criterios que emergieron en el discurso de todos los profesores entrevistados fueron: contexto que los problemas se adecuen al contexto de los estudiantes- e interés o atractivo del problema -desde el punto de vista afectivo/emocional de los estudiantes-. Sin embargo, en las entrevistas se evidencias algunos rasgos que hacen pensar que tales dos criterios han sido adquiridos y considerados por los profesores, a partir de las políticas educativas y propuestas ministeriales. Así, una pregunta que podríamos plantearnos para una investigación futura es: ¿el tipo de tareas propuestas por los profesores tributan al desarrollo de nuevas habilidades, actitudes y competencias de los estudiantes? O la emergencia de tales criterios (contexto e interés) sólo es discurso que busca hacer presentes las solicitudes hechas por el Ministerio de Educación de Chile. Además, cuando los profesores señalaban contexto como uno de los criterios para seleccionar o plantear problemas, siempre lo consideraban desde el punto de vista de la "aplicación a la vida real", es decir, desde un punto de vista extra matemático. Malaspina (2017) señala que cuando se diseña un problema por variación o elaboración, el contexto del mismo puede ser tanto intra-matemático como extra matemático, dependiendo del énfasis que se le quiera dar al problema, aspecto que parece no tener en cuenta los profesores.

Tabla 2

\section{Criterios utilizados por los profesores para elegir problemas para sus clases}

Por elaboración Planteamiento de problemas (creación)

- Atractivo del problema. Por variación (adaptación)

Contexto de los estudiantes. - Contexto de los estudiantes

- Que promuevan el razonamien- to matemático.

- Que posean una estructura de matematización (diversas estrategias, representaciones, etc.).
Selección de problemas

Ya creados en otras fuentes (Repetición)

Nota: Fuente propia de la investigación

Luis R. Pino-Fan, Daniela Ilse Báez-Huaiquián, Juan G. Molina-Cabero y Elizabeth Hernández-Arredondo Artículo protegido por licencia Creative Commons: BY-NC-ND / Protected by Creative Commons: BY-NC-ND. 
Otro elemento presente en las respuestas de los profesores fue la necesidad de cumplir con las expectativas de la comunidad, la región, entre otros. Un ejemplo de ello es cuando manifiestan a veces que su trabajo (prácticas de planificar y resolver problemas) queda condicionado con el uso de un determinado libro de texto o de cierto tipo de problemas exigidos por la institución, para alcanzar los resultados en alguna prueba estandarizada. Lo anterior evidencia que, en muchos casos, la propuesta de problemas que se llevan al aula queda supeditadas a una serie de expectativas institucionales, que poco tienen que ver con los aprendizajes y comprensión de las matemáticas por parte de los estudiantes, lo cual deja en segundo plano lo pretendido por el currículo o el perfil de egreso.

Un elemento señalado por los profesores, como agente que obstaculiza el desarrollo de la actividad de diseñar problemas (por creación o variación), es la sobre carga administrativa por parte de los centros de trabajo, lo cual, a su vez, contribuye con la falta de espacios de reflexión para los profesores, sobre sus prácticas profesionales y sobre actividades como el diseño de problemas. Este hecho es importante debido a que, sin la reflexión de los profesores sobre su práctica, la resolución de problemas queda centrada en la 'capacidad' de solución de los estudiantes, perdiéndose así oportunidades valiosas para identificar potenciales mejoras en las prácticas de plantear y resolver problemas en el aula -a través de buenos problemas y buenas preguntas que permitan potenciar el pensamiento matemáticos de los estudiantes-, aspectos que son pretendidos por el currículo de matemáticas chileno.

De acuerdo con algunos profesores entrevistados, principalmente con quienes diseñan problemas por creación o variación, la creación de espacio para reflexionar sobre la propia práctica, en concreto sobre el tipo de problemas planteados en clases y las estrategias que se adoptan con los estudiantes para su solución, permitiría incluso potenciar competencias profesionales clave para el profesor de matemáticas. Al respecto, algunos trabajos sobre conocimientos didáctico-matemáticos y competencias profesionales del profesor de matemáticas (Castro, Pino-Fan \& Velásquez-Echavarría, 2018; Pino-Fan, Font \& Breda, 2017) sugieren que las competencias clave del profesor de matemática son dos: competencia matemática y competencia de análisis e intervención didáctica. Además, desde el modelo CCDM, se sugiere que las competencias que requiere el profesor para llevar a cabo la actividad de plantear buenos problemas en el aula y ayudar a sus estudiantes a resolverlos con el planteamiento de buenas preguntas, deambulan entre la competencia matemática y la competencia de análisis didáctico, cuyas sub-competencias son: i) competencia para resolver problemas (profesor como resolutor); ii) competencia para diseñar problemas; y iii) competencia para analizar la actividad matemática de los estudiantes o la propia.

Los criterios propuestos por los profesores de nuestro estudio, en su mayoría, fueron relativos a la componente epistémica (i.e., relativas a la riqueza matemática), y algunos aspectos ecológicos (relativos al contexto), cognitivos (relativos a los conocimientos previos de los estudiantes, nivel de demanda cognitiva del problema, etc.) y afectivos (motivación y generación de interés en los estudiantes). Este es un resultado teórico-metodológico relevante, pues evidencia que los profesores de nuestro estudio utilizan implícitamente los criterios y descriptores propuestos por el modelo CCDM 
(Breda, Pino-Fan \& Font, 2017; Castro, Pino-Fan \& Velásquez-Echavarría, 2018; Morales-López \& Font, 2019) para reflexionar y valorar la propia práctica.

\section{Agradecimientos}

Este trabajo ha sido desarrollado parcialmente en el marco del Proyecto de Investigación Fondecyt 1200005 financiado por la Agencia Nacional de Investigación y Desarrollo (ANID) de Chile

\section{Referencias}

Arcavi, A., \& Friedlander, A. (2007). Curriculum developers and problem solving: the case of Israeli elementary school projects. ZDM Mathematics Education, 39(5-6), 355-364. doi: https://doi.org/10.1007/s11858-007-0050-3

Borasi, R. (1986). On the nature of problems. Educational Studies in Mathematics, 17(2), 125-141. doi: https://doi.org/10.1007/BF00311517

Breda, A.; Pino-Fan, L., \& Font, V. (2017). Meta didactic-mathematical knowledge of teachers: criteria for the reflection and assessment on teaching practice. Eurasia Journal of $\mathrm{Ma}$ thematics, Science and Technology Education, 13(6), 1893-1918. doi: https://doi. org/10.12973/eurasia.2017.01207a

Breda, A., Font, V., \& Pino-Fan, L. (2018). Criterios valorativos y normativos en la didáctica de las matemáticas: El caso del constructo idoneidad didáctica. Bolema, Río Claro, 32(60), 255-278. doi: http://dx.doi. org/10.1590/1980-4415v32n60a13

Castro, W. F., Pino-Fan, L., \& Velásquez-Echavarría, H. (2018). A proposal to enhance preservice teacher's noticing. Eurasia Journal of Mathematics, Science and Technology Education, 14(11), 1-17. doi: https://doi.org/10.29333/ ejmste/92017

Cohen, L., Manion, L., \& Morrison, K. (2011). Research Methods in Education (7th ed.). London: Routledge.

Crespo, S. (2003). Learning to pose mathematical problems: exploring changes in preservice teachers' practices. Educational Studies in
Mathematics, 52(3), 243-270. doi: https://doi org/10.1023/A:1024364304664

De Guzmán, M. (1993). Tendencias innovadoras en educación matemática. Madrid, España: Universidad Complutense. Recuperado de http://nautilus.fis.uc.pt/bspm/revistas/25/009-034.150.pdf

Felmer, P., Pehkonen, E., \& Kilpatrick, J. (2016). Posing and solving mathematical problems. Suiza: Springer International Publishing. Recuperado de https://link.springer.com/book/1 0.1007\%2F978-3-319-28023-3

Felmer, P., \& Perdomo-Díaz, J. (2016). Novice Chilean Secondary Mathematics Teachers as Problem Solvers. In P. Felmer, E. Pehkonen, \& J. Kilpatrick (2016), Posing and solving mathematical problems (pp. 287-308). Suiza: Springer International Publishing. doi: https://doi.org/10.1007/978-3-319-28023-3_17

Font, V., Planas, N., \& Godino, J. D. (2010). Modelo para el análisis didáctico en educación matemática. Infancia y Aprendizaje, 33(1), 89-105. Recuperado de https://www.academia.edu/1252367/Modelo_para_el_an\%C3\%A1lisis_did\%C3\%A1ctico_en_educaci $\% \mathrm{C} 3 \% \mathrm{~B} 3 \mathrm{n} \_$matem $\% \mathrm{C} 3 \% \mathrm{~A} 1$ tica

Font, V. (2011). Competencias profesionales en la formación inicial de profesores de matemáticas de secundaria. Unión. Revista Iberoamericana de Educación Matemática, 26, 9-25. Recuperado de http://www.fisem.org/www/ union/revistas/2011/26/archivo_5_de_volumen_26.pdf

Font, V., Godino, J. D., \& Gallardo, J. (2013). The Emergence of Objects from Mathematical Practices. Educational Studies in $\mathrm{Ma}$ thematics, 82(1), 97-124. doi: https://doi. org/10.1007/s10649-012-9411-0

Foster, C. \& Inglis, M. (2017). Teachers' appraisals of adjectives relating to mathematics tasks. Educational Studies in Mathematics, 95(3), 283-301. https://doi.org/10.1007/ s10649-017-9750-y

Godino, J. D., Batanero, C., \& Font, V. (2007). The onto-semiotic approach to research in mathematics education. ZDM Mathematics Education, 39(1-2), 127-135. doi: https://doi. org/10.1007/s11858-006-0004-1

Godino, J. D., Batanero, C., \& Font, V. (2019). The onto-semiotic approach: implications for the prescriptive character of didactics. For the Learning of Mathematics, 39(1), 37- 42. 
Godino, J. D., Giacomone, B., Font, V., \& Pino-Fan, L. (2018). Conocimientos profesionales en el diseño y gestión de una clase sobre semejanza de triángulos. Análisis con herramientas del modelo CCDM. Avances de Investigación en Educación Matemática, 13, 63-83. Recuperado de https://www.aiem.es/index.php/aiem/ article/view/224

Halmos, P. R. (1980). The heart of mathematics. The American Mathematical Monthly, 87(7), 519524. Recuperado de https://www.jstor.org/ stable/2321415

Kaiber, C. T., Lemos, A., \& Pino-Fan, L. (2017). Enfoque ntossemiótico do Conhecimento e da Instrução Matemática (EOS): um panorama das pesquisas na América Latina. Perspectivas da Educação Matemática, 10(23), 531 -552. Recuperado de https://periodicos.ufms.br/index.php/pedmat/article/ download/5056/4091

Kilpatrick, J. (1985). A Retrospective Account of the Twenty-five Years of Research on Teaching Mathematical Problem Solving. En E. A. Silver (Ed.), Teaching and learning mathematical problem solving: multiple research perspective (pp.1-15). Hillsdale, USA: Lawrence Erlbaum.

Klinshtern, M., Koichu, B., \& Berman, A. (2015). What do high school teachers mean by saying "I pose my own problems". In F. M., Singer, N., Ellerton \& J., Cai. (2015). Mathematical Problem Posing. From research to effective practice (pp. 449-467). New York: Springer. doi: https://doi. org/10.1007/978-1-4614-6258-3_22

Liljedahl, P., Santos-Trigo, M., Malaspina, U., \& Bruder, R. (2016). Problem solving in Mathematics Education. Hamburg, Germany: ICME-13 Topical Surveys, SpringerOpen. doi: https:// doi.org/10.1007/978-3-319-40730-2_1

Love, E., \& Pimm, D. (1996). 'This is so': a text on texts. En A. Bishop, K. Clements, C. Keitel, J. Kilpatrick, y C. Laborde (Eds.), International handbook of mathematics education (pp. 371-409). Dordrecht, Holanda: Kluwer. Recuperado de https://link.springer.com/ chapter/10.1007/978-94-009-1465-0_11

Malaspina, U., Mallart, A., \& Font, V. (2015). Development of teachers' mathematical and didactic competencies by means of problem posing. En K. Krainer y N. Vondrová (Eds.), Proceedings of the Ninth Congress of the European Society for Research in Mathematics Education (pp. 2861-2866). Prague, Czech Republic: CERME. Recuperado de https:/hal.archives-ouvertes.fr/hal-01289630/document

Malaspina, U. (2016). Creación de problemas: Sus potencialidades en la enseñanza y aprendizaje de las matemáticas. Cuadernos de Investigación y Formación en Educación Matemática, 11(15), 321-331. Recuperado de https:// revistas.ucr.ac.cr/index.php/cifem/article/ view/23946/24101

Malaspina, U. (2017). La creación de problemas como medio para potenciar la articulación de competencias y conocimientos del profesor de matemáticas. En J. M. Contreras, P. Arteaga, G. R. Cañadas, M. M. Gea, B. Giacomone y M. M. López-Martín (Eds.), Actas del Segundo Congreso International Virtual sobre el Enfoque Ontosemiótico del Conocimiento y la Instrucción Matemáticos. Pontificia Universidad Católica del Perú, Perú. Recuperado de http://enfoqueontosemiotico.ugr.es/ civeos/malaspina.pdf

Morales-López, Y. \& Font, V. (2019). Evaluation by a teacher of the suitability of her mathematics class. Educação e Pesquisa, 45, 1-19. doi: https://dx.doi.org/10.1590/ s1678-4634201945189468

NCTM. (1989). Curriculum and Evaluation Standards for School Mathematics. Reston, Virginia, USA: NCTM. Recuperado de http:// csmc.missouri.edu/PDFS/CCM/summaries/ standards_summary.pdf

NCTM. (2016). De los principios a la acción. Para garantizar el éxito matemático para todos. Reston, Virginia, USA: NCTM.

Perdomo-Díaz, J., Felmer, P., Randolph, V., González, G. (2017). Problem Solving as a Professional Development Strategy for Teachers: a Case Study with Fractions. Eurasia Journal of Mathematics, Science and Technology Education, 13(3), 987-999. doi: https://doi. org/10.12973/eurasia.2017.00653a

Pino-Fan, L., Assis, A., \& Castro, W. F. (2015). Towards a methodology for the characterization of teachers' didactic-mathematical knowledge. Eurasia Journal of Mathematics, Science \& Technology Education, 11(6), 1429-1456. Recuperado de https:// pdfs.semanticscholar.org/dea9/70789e49dbe1d8206a04b19f4096fd186b89.pdf 
Pino-Fan, L., \& Godino, J. D. (2015). Perspectiva ampliada del conocimiento didáctico-matemático del profesor. PARADIGMA, 36(1), 87-109. Recuperado de https://pdfs.semanticscholar.org/bb35/

Pino-Fan, L., Font, V., \& Breda, A. (2017). Mathematics teachers' knowledge and competences model based on the onto-semiotic approach. In B. Kaur, W. K. Ho, T. L. Toh \& B. H. Choy (Eds.), Proceedings of the 41st Conference of the International Group for the Psychology of Mathematics Education (pp. 33-40). Singapure, Asia: PME. Recuperado de https://www. dropbox.com/s/6t1yfkuzy0ynbb4/Pino-Fan,Font \%26 Breda_CCDM_PME.pdf?dl=0

Pino-Fan, L. Godino, J. D., \& Font, V. (2018). Assessing key epistemic features of didactic-mathematical knowledge of prospective teachers: the case of the derivative. Journal of Mathematics Teacher Education, 21(1), 63-94. doi: http://dx.doi.org/10.1007/ s10857-016-9349-8

Pino-Fan, L., Guzmán, I., Larraín, M., \& Vargas, ( (2018). La formación inicial de profesor en Chile: 'voces' de la comunidad chiler de investigación en educación matemátic
Uniciencia, 32(1), 68-88. doi: http://dx.doi. org/10.15359/ru.32-1.5

Polya, G. (1986). How to Solve it. [Cómo plantear y resolver problemas]. New Jersey, USA: Princeton University Press.

Presmeg, N. (2014). Visualization and Learning in Mathematics Education. In S. Lerman (Ed.), Encyclopedia of Mathematics Education. Dordrecht, Holanda: Springer. doi: https:// doi.org/10.1007/978-94-007-4978-8

Ron, G., Zaslavsky, O. \& Zodik, I. (2013). Engaging teachers in the web of considerations underlying the design of tasks that foster the need for new mathematical concept tools. In C. Margolinas (Ed.), Task design in mathematics education; Proceedings of the International Commission on Mathematical Instruction Study 22, (pp. 641-647), Oxford, UK. Recuperado de http://hal.archives-ouvertes.fr/ hal-00834054

Schoenfeld, A. H. (1985). Mathematical problem solving. New York: Academic Press. doi: https:// doi.org/10.1016/C2013-0-05012-8

inger, F.M., Ellerton, N., \& Cai, J. (2015). Mathematical Problem Posing. New York: Springer. doi: 10.1007/978-1-4614-6258-3

\section{(2) $(1) \Theta$}

Criterios utilizados por profesores de matemáticas para el planteamiento de problemas en el aula (Luis R. Pino-Fan, Daniela Ilse Báez-Huaiquián, Juan G. Molina-Cabero y Elizabeth Hernández-Arredondo) in Uniciencia is protected by Attribution-NonCommercialNoDerivs 3.0 Unported (CC BY-NC-ND 3.0) 


\section{Anexo}

\section{Cuestionario}

Estimado profesor, primeramente, agradecer su disposición y tiempo para poder desarrollar esta actividad. Este cuestionario es parte de una investigación que tiene por objetivo comprender cómo los profesores de matemáticas seleccionan los problemas para su práctica de enseñanza. Ninguna de las preguntas tiene una respuesta "correcta" o "incorrecta". Es muy importante para nuestra investigación el que responda todas las preguntas de forma honesta. Se solicitan los datos del encuestado con motivos meramente investigativos, sus datos serán guardados digitalmente para llevar un orden en cada una de las etapas del proceso, y se les garantiza, en todo momento, el anonimato absoluto.

\section{Datos personales}

Nombre:

Nivel educativo en el que se desempeña actualmente:

2. ¿Cuántos años de experiencia usted tiene enseñando matemáticas?

\begin{tabular}{|l|l|}
\hline $1-2$ años & \\
\hline $3-5$ años & \\
\hline $6-10$ años & \\
\hline Más de 10 años & \\
\hline
\end{tabular}

3. Generalmente, ¿Cuál es el nivel de complejidad que impone usted en sus clases de matemáticas?

\begin{tabular}{|l|l|}
\hline Alto & \\
\hline Medio & \\
\hline Bajo & \\
\hline
\end{tabular}

4. ¿Hasta qué punto utiliza los siguientes recursos para seleccionar problemas matemáticos útiles para su práctica de enseñanza? Considere el siguiente orden numérico para definir su respuesta.

1. Casi siempre

2. A menudo

3. A veces

4. Raramente

5. Casi nunca

\begin{tabular}{|l|l|l|l|l|l|}
\hline & $\mathbf{1}$ & $\mathbf{2}$ & $\mathbf{3}$ & $\mathbf{4}$ & $\mathbf{5}$ \\
\hline Libros de texto escolar & & & & & \\
\hline Otros libros & & & & & \\
\hline Recursos de internet & & & & & \\
\hline Talleres de desarrollo profesional & & & & & \\
\hline Compañeros profesores, colegas & & & & & \\
\hline Mis estudios académicos anteriores (notas, apuntes, etc.) & & & & & \\
\hline Plantear mis propios problemas & & & & & \\
\hline Problemas planteados por los alumnos & & & & & \\
\hline Otros & &
\end{tabular}


Si informó otros recursos, por favor, indique todos aquellos que utilice:

5. ¿En qué medida ocurren las siguientes situaciones en su práctica de enseñanza? Considere el siguiente orden numérico para dar su respuesta.

1. Casi siempre

2. A menudo

3. A veces

4. Raramente

5. Casi nunca

\begin{tabular}{|l|c|c|c|c|c|}
\hline & $\mathbf{1}$ & $\mathbf{2}$ & $\mathbf{3}$ & $\mathbf{4}$ & $\mathbf{5}$ \\
\hline Uso mis propios problemas & & & & & \\
\hline Motivo a los estudiantes a plantear sus propios problemas & & & & & \\
\hline Promuevo la discusión en clase & & & & & \\
\hline Fomento el trabajo en grupo. & & & & & \\
\hline
\end{tabular}

6. Si usted plantea sus propios problemas para enseñar clases de matemáticas, ¿por qué lo hace?, ¿con qué propósito plantea sus propios problemas?

7. En qué medida las siguientes situaciones son de su responsabilidad:

\begin{tabular}{|l|l|l|}
\hline & Poco & Mucho \\
\hline Planificación del año escolar & & \\
\hline Planificación de cada una de las lecciones & & \\
\hline Ejecución de mi planificación & & \\
\hline Selección de los problemas matemáticos a utilizar. & & \\
\hline
\end{tabular}

8. ¿Cuál es su formación profesional y último grado académico? (e.g., Licenciado en Matemáticas, Magister en Educación Matemática).

9. ¿Podríamos contactarnos próximamente con la finalidad de hacerle una entrevista?

10. ¿Está usted interesado en recibir actualizaciones sobre los resultados de estudio?

\begin{tabular}{|l|l|}
\hline Sí & \\
\hline No & \\
\hline
\end{tabular}

